\title{
Bilobalide inhibits 6-OHDA-induced activation of NF-kB and loss of dopam- inergic neurons in rat substantia nigra ${ }^{1}$
}

\author{
Ling-yun LI, Xi-lin ZHAO, Xi-feng FEI, Zhen-lun GU, Zheng-hong QIN, Zhong-qin LIANG ${ }^{2}$ \\ Department of Pharmacology, Soochow University School of Medicine, Laboratory of Aging and Nervous Diseases, Soochow University \\ School of Medicine, Suzhou 215123, China
}

\section{Key words}

bilobalide; 6-hydroxydopamine; Parkinson's disease; NF- $\mathrm{BB}$; apoptosis

${ }^{1}$ Project supported by grants from the National Natural Science Foundation of China (No 30672452), the Natural Science Foundation of Jiangsu Province (№ BK2006051), and the Natural Science Foundation of Jiangsu High Education Bureau (No 05KJB3 10117). ${ }^{2}$ Correspondence to Prof Zhong-qin LIANG. Phn 86-512-6588-0119.

Fax 86-512-6588-0406.

E-mail liangzhongqin@suda.edu.cn

Received 2007-10-21

Accepted 2008-02-18

doi: $10.1111 / \mathrm{j} .1745-7254.2008 .00787 . x$

\begin{abstract}
Aim: To investigate the effects of bilobalide on the activation of NF- $\mathrm{KB}$, and apoptosis of dopaminergic neurons induced by 6-hydroxydopamine (6-OHDA). Methods: A rat model of Parkinson's disease was produced with a unilateral infusion of 6-OHDA $(8 \mu \mathrm{g})$ into the substantia nigra par compact. Bilobalide was administered 5,10 , and $20 \mathrm{mg} / \mathrm{kg}$ (ip) once a day for $7 \mathrm{~d}$, starting $6 \mathrm{~d}$ prior to the 6 OHDA infusion. The rats were subjected to locomotor activity and rotational behavior testing 2 or 3 weeks after the 6-OHDA infusion. The expressions of tyrosine hydroxylase $(\mathrm{TH})$ and NF- $\mathrm{\kappa B}$ p 65 were examined by immunofluorescence. The loss of dopaminergic neurons was detected by Nissl's staining. Terminal deoxynucleotidyl transferase-mediated dUTP nick-end labeling was used to identify apoptosis. Results: The behavioral changes due to 6-OHDA were significantly restored by bilobalide pretreatment. Bilobalide inhibited the 6-OHDAinduced loss of TH-positive neurons, decreased the activation of NF- $\mathrm{KB}$, and protected dopaminergic neurons from apoptosis remarkably. Conclusion: NF- $\kappa B$ activation contributes to the 6-OHDA-induced loss of dopaminergic neurons, and the inhibition of the NF- $\mathrm{KB}$ pathway is likely to be involved in the neuroprotective effect of bilobalide.
\end{abstract}

\section{Introduction}

Parkinson's disease (PD) is a motor function disorder caused primarily by the loss of the neurotransmitter dopamine (DA) in the central nervous system (CNS) as a result of selective degeneration of nigral dopaminergic neurons $\mathrm{s}^{[1-3]}$. It is generally believed that both genetic and environmental factors play roles in PD, but the exact mechanisms of neuronal death have not been fully understood. Several scientific reports demonstrate that apoptosis of the neurons in the substantia nigra ( $\mathrm{SN}$ ) plays an important role in the development of PD. A number of studies suggest that oxidative stress contributes to dopaminergic neuron degeneration. In previous studies, we found that the transcription factor $\mathrm{NF}-\kappa \mathrm{B}$ plays a pro-apoptotic role in the excitotoxin-induced apoptotic death of neurons in the SN par compact (SNpc), possibly through upregulating p53 and c-Myc ${ }^{[4-6]}$. Increased $\mathrm{NF}-\kappa \mathrm{B}$ immunoreactivity has been reported in the SNpc of patients with $\mathrm{PD}$, which suggests that $\mathrm{NF}-\kappa \mathrm{B}$ plays a role in oxidative stress-induced dopaminergic neuron degeneration in $\mathrm{PD}^{[7]}$

Although various treatments are successfully used to alleviate the symptoms of PD, none of them prevents or halts the neurodegenerative process of the disease. Clinical studies have shown that ginkgo extracts, which have been widely used as a dietary supplement in the United States, exhibit several beneficial effects in a variety of CNS disorders, including Alzheimer's disease (AD) and PD ${ }^{[8-11]}$.

The active components of Ginkgo biloba are considered to be the ginkgo flavone glycosides, the polycyclic lactone ginkgolides A, B, C, J, and bilobalide ${ }^{[12]}$. EGb 761, a standard extract of Ginkgo biloba (EGb) with potent antioxidant properties, shows great benefits on the CNS, being able to enhance peripheral and cerebral circulation ${ }^{[13]}$, and protecting neurons against a variety of insults. 
Several studies have reported that EGb exerts beneficial effects in AD and PD models. A recent study indicated that that administration of $\mathrm{E} G b 761$ can reduce $\beta$-amyloid oligomers and restore cAMP respond element binding protein (CREB) phosphorylation in the hippocampus of a transgenic mouse model of $\mathrm{AD}^{[14]}$. EGb pretreatment is able to reverse $\beta$-amyloid-peptide-induced isoprostane production in the rat brain in vitro ${ }^{[15]}$ and inhibit cerebral monoamine oxidase (MAO) activity in vivo ${ }^{[16]}$. Wu and $\mathrm{Zhu}^{[17]}$ demonstrated that EGb could attenuate 1-methyl-4-phenyl-1,2,3,6tetrahydropyridine (MPTP)-induced nigrostriatal dopaminergic neurotoxicity in C57 mice. Another study suggested that $\mathrm{E} G b$ offers dose-dependent protection against 6hydroxydopamine (6-OHDA)-induced parkinsonism in rats. The pretreatment of rats with EGb leads to a restoration of compromised behavioral activity, levels of tyrosine hydroxylase $(\mathrm{TH})$, and neurotransmitter $\mathrm{DA}^{[18]}$. Cao et al ${ }^{[19]}$ found that the combined use of EGb with levodopa reduces the toxic effects of levodopa, and thus may be a therapeutic strategy in the management of motor function in PD.

Bilobalide, a sesquiterpene lactone, which constitutes of approximately $3 \% \mathrm{EGb} 761$, is quantitatively the major single chemical constituent of EGb 761. The first pharmacological action detected was a beneficial effect on cytotoxic brain edema caused by triethyltin ${ }^{[20]}$. Bilobalide has now been demonstrated to inhibit delayed ischemic neuronal death and reduce infarct volume after focal cerebral ischemia and ischemia-induced neuronal damage in rodents ${ }^{[21-24]}$. Zhou et $a l^{[25]}$ demonstrated that bilobalide could protect against $\beta$ amyloid toxicity and reactive oxygen species (ROS)-induced apoptosis in PC12 cells. It has also been demonstrated that bilobalide has potent inhibitory actions on the $N$-methyl-Daspartate-induced activation of phospholipase $\mathrm{A}_{2}$ and the associated phospholipid breakdown in the brain ${ }^{[26]}$.

These studies suggest that bilobalide is a main active component in the neuroprotective actions of $\mathrm{E} G b$, but it has not been reported whether bilobalide has a protective effect on the neurodegeneration in PD or not. In the present study, we explored the neuron protective effects of bilobalide in a rat model of PD induced by 6-OHDA. We report that neuron pathology and behavioral changes can be restored effectively by pretreatment with bilobalide, suggesting that bilobalide may be a candidate to alleviate the Parkinsonrelated pathology.

\section{Materials and methods}

Animals Male Sprague-Dawley rats were obtained from the Center for Experimental Animals, Soochow University (Suzhou, China), and weighed 250-280 g at the start of the experiment. The rats were housed under standardized light/ dark cycle (12 h) conditions with access to food and water ad libitum. All procedures were performed in accordance with the NIH Guidelines for the Care and Use of Laboratory Animals.

Treatment with bilobalide Bilobalide was provided by Zhong-liang CHEN (Department of Phytochemistry, Shanghai Institute of Materia Medica, Chinese Academy of Sciences, Shanghai, China), and the purity of this compound was $98 \%$ (HPLC). Bilobalide was dissolved in DMSO and then diluted with $0.9 \%$ sodium chloride. The final concentration of DMSO was $1 \%(v / v)$. Bilobalide was administered by intraperitoneal injection (ip) to rats once daily at doses of 5,10 , and $20 \mathrm{mg} \cdot \mathrm{kg}^{-1} \cdot \mathrm{d}^{-1}$ for 7 successive days, respectively.

PD model induced by 6-OHDA After $7 \mathrm{~d}$ of bilobalide treatment, the rats were anesthetized with $400 \mathrm{mg} / \mathrm{kg}$ chloral hydrate (ip), and then placed on a stereotaxic instrument (Stoelting, Wood Dale, IL, USA). A hole was drilled through the skull to the top of the dura, and a 29 gauge stainless steel needle was lowered to the left SNpc (coordinates: $-5.2 \mathrm{~mm}$ from bregma, $2.1 \mathrm{~mm}$ from midline, $7.8 \mathrm{~mm}$ from surface). A solution of 6-OHDA with $0.1 \%$ ascorbic acid-saline (Sigma, St Louis, MO, USA) was dissolved in $0.9 \% \mathrm{NaCl}(w / v)$ to a concentration of $4 \mu \mathrm{g} / \mu \mathrm{L}$, and then infused unilaterally into the left SNpc by a microinfusion pump delivering $2 \mu \mathrm{L}(8 \mu \mathrm{g})$ over a 5 min period. After infusion, the needle was kept in place for $5 \mathrm{~min}$ to ensure diffusion. Sham-operated animals received an equal volume of $0.9 \% \mathrm{NaCl}$ delivered by the same method.

Animal grouping 1 The rats were divided into 6 groups, each consisting of 10 animals. Group 1 included sham-operated rats pretreated with vehicle (S); group 2 included shamoperated rats pretreated with $20 \mathrm{mg} / \mathrm{kg}$ bilobalide $(\mathrm{S}+\mathrm{H}-\mathrm{BB})$; group 3 included PD model rats pretreated with vehicle (M); group 4 included PD model rats pretreated with $5 \mathrm{mg} / \mathrm{kg}$ bilobalide $(\mathrm{M}+\mathrm{L}-\mathrm{BB})$; group 5 included $\mathrm{PD}$ model rats pretreated with $10 \mathrm{mg} / \mathrm{kg}$ bilobalide $(\mathrm{M}+\mathrm{M}-\mathrm{BB})$; and group 6 included PD model rats pretreated with $20 \mathrm{mg} / \mathrm{kg}$ bilobalide $(\mathrm{M}+\mathrm{H}-\mathrm{BB})$. The animals were used to evaluate the effect of pretreatment with bilobalide on the behavioral changes and neuron survival in 6-OHDA-lesioned rats.

Animal grouping 2 The rats were divided into 3 groups, each consisting of 10 animals. Group 1 included sham-operated rats pretreated with vehicle (control); group 2 included PD model rats pretreated with vehicle (6-OHDA); and group 3 included $P D$ model rats pretreated with $10 \mathrm{mg} / \mathrm{kg}$ bilobalide (6-OHDA+M-BB). These animals were used to explore the mechanism involved in the effects of bilobalide in vivo.

Behavior studies On d 14 after a stereotaxic injection of 
6-OHDA, the motor activity of the animals was tested for locomotor activity in a computerized animal activity video analyzer (Shanghai Jiliang Software Technology, Shanghai, China). Each rat was placed in the chamber and its locomotor activity was monitored by the activating camera and viewed on the screen. The activities of the animals at $5 \mathrm{~min}$ periods were recorded, and the data of the locomotion time were collected by individuals who were trained in behavioral observation.

On d 14 and 21 after infusion of 6-OHDA, the animals were subject to rotational behavior testing ${ }^{[27]}$. The rats were administered R-(2)-apomorphine hydrochloride $(0.5 \mathrm{mg} / \mathrm{kg}$ in $0.1 \%$ ascorbic acid-saline, subcutaneously) and placed in a transparent cylindrical cage. Contralateral rotations $\left(360^{\circ}\right.$, in short axis) over a $30 \mathrm{~min}$ interval from the initiation of rotation were recorded.

Immunofluorescence of the brain sections Twenty four days after the infusion of 6-OHDA, the rats were anaesthetized and then perfused transcardially with $200 \mathrm{~mL}$ precooled $0.01 \mathrm{~mol} / \mathrm{L}$ phosphate-buffered saline (PBS; $\mathrm{pH} 7.4$ ), followed by $200 \mathrm{~mL}$ perfusate containing $4 \%$ paraformaldehyde in 0.1 mol/L phosphate buffer ( $\mathrm{PB}, \mathrm{pH} 7.4)$. The brains were postfixed overnight in the same paraformaldehyde fixative and then transferred to $20 \%$ sucrose solution until they sank to the bottom of the containers. The brains were snap frozen and sectioned at $30 \mathrm{~mm}$ thickness with a cryostat (Leica Microsystems GmbH, Wetzlar, Germany). Free-floating sections were washed in $0.01 \mathrm{~mol} / \mathrm{L}$ PBS 3 times for $10 \mathrm{~min}$ each and incubated in PBS with $0.1 \%$ Triton $\mathrm{X}-100$ for $1 \mathrm{~h}$ at room temperature (RT).The brain sections were then blocked with $1 \%$ bovine serum albumin (BSA) for $1 \mathrm{~h}$ at RT and then incubated with mouse monoclonal antibody TH (1:3000; Sigma, USA), a primary antibody recognizing $\mathrm{TH}$, in PBS containing $0.1 \%$ Triton $\mathrm{X}-100$ at $4{ }^{\circ} \mathrm{C}$ for $48 \mathrm{~h}$. Sections were subsequently rinsed in PBS and incubated for $1 \mathrm{~h}$ with the secondary antibody (Cy3-conjugated donkey antimouse immunoglobulin G [IgG]; Jackson ImmunoResearch, West Grove, PA, USA). Sections were mounted on glass slides, coverslipped with antifade mountant, and then observed with a fluorescence microscope (Nikon, Tokyo, Japan).

Nissl's staining The brain sections were stained with $0.75 \%$ cresyl violet, dehydrated twice through graded alcohols $(70 \%, 95 \%$, and $100 \%)$, cleared in xylenes 3 times for 5 min each, coverslipped with resinous mountant, and then observed with a light microscope.

Double immunofluorescence Approximately 6-24 h after the 6-OHDA infusion, the rats were perfused, and the brains were then frozen and sectioned according to the protocol described earlier. The brain sections were rinsed with
PBS, incubated in PBS with $0.1 \%$ Triton X-100, blocked with $1 \%$ BSA in PBS, and then incubated with primary and secondary antibodies sequentially. To examine if the activation of NF- $\kappa \mathrm{B}$ p65 occurs in nigral neurons, the brain sections were incubated with a mouse monoclonal antibody against TH (1:3000) and rabbit polyclonal antibody against NF- $\kappa B$ p65 (1:500; Chemicon, Temecula, CA, USA), and then incubated with the secondary antibodies (fluoresceinisothiocyanate [FITC]-conjugated donkey antimouse IgG, 1:1000 and Cy3-conjugated donkey antirabbit IgG, 1:1000). Sections were washed in PBS, mounted on glass slides, coverslipped, and then examined with a laser confocal system (Leica Microsystems GmbH, Wetzlar, Germany).

Terminal deoxynucleotidyl transferase-mediated dUTP nick-end labeling The low molecular weight DNA fragments as well as the single strand breaks ("nicks") in high molecular weight DNA can be identified by labeling free 3'-OH termini with modified nucleotides by an enzymatic reaction with terminal deoxynucleotidyl transferase, which catalyzes polymerization of nucleotides to free 3'-OH DNA ends in a template-independent manner. This method labels these hydroxyl groups with fluorescein-conjugated deoxynucleotides. Fluorescein generates an intense signal that can be detected by a fluorescence microscope.

Twenty four hours after the 6-OHDA infusion, the rat brain sections were prepared according to the protocol described earlier. The brain sections were incubated with a mouse monoclonal antibody against $\mathrm{TH}$, and reacted with a secondary antibody (Cy3-conjugated donkey antimouse IgG). Then the brain sections were mounted on polylysine-coated glass slides. DNA damage was detected using a Fluorescein FragEL DNA fragmentation detection kit (Calbiochem, San Diego, CA, USA) according to the protocol of the manufacturer. The sections were then washed in PBS, counterstained with 4',6'-diamidino-2-phenylindole dihydrochloride (DAPI), and coverslipped. To determine the relationship between NF- $\kappa B$ p65 and DNA damage, the brain sections were incubated with rabbit polyclonal antibodies against NF- $\mathrm{B}$ p65, and subsequently reacted with secondary antibodies (Cy3-conjugated donkey antirabbit IgG). DNA damage was then detected as described earlier. Slides were observed with a laser confocal system.

Cell counting Staining with a TH antibody can delineate the SNpc in coronal sections. The sections were immunofluorescent labeled for TH. Stained cells were counted within the outlines, and total estimates were obtained. Labeled profiles were counted only if the first recognizable profile of the cell soma came into focus within the counting frame ${ }^{[28]}$. Using every coronal section, the analysis was performed 
starting with the first appearance of TH-positive neurons, extending to the most caudal parts of the SNpc and including both hemispheres. Sections were viewed under an inverted fluorescence microscope. Each group included 6 rats, and 6 brain sections from each rat were counted.

An estimation of the percentages of TH-positive neurons having undergone recombination with $\mathrm{NF}-\kappa \mathrm{B}$ p 65 was obtained by montage images (FITC and Cy3) of the left SNpc under a laser confocal system. The total number of THpositive neurons and total number of $\mathrm{TH} / \mathrm{NF}-\kappa \mathrm{B}$ doublepositive neurons were counted for each section. The number of double-labeled neurons divided by the total number of TH-positive neurons was the percentage of recombination, which showed the activation of NF- $\kappa \mathrm{B}$ in each group. Each group included 6 rats, and 6 brain sections from each rat were counted.

Statistical analysis Data are presented as mean \pm SEM. One-way ANOVA or the Student's unpaired two-tailed test was used for the statistical analysis. Statistical significance was set at $P<0.05$.

\section{Results}

Bilobalide restored 6-OHDA-induced impairment on motor activity In the PD model group, the motor activity was reduced significantly as compared to the sham-operated group $(\mathrm{S})$. The time spent on locomotion was significantly decreased $(76.9 \%)$. Different doses of bilobalide (L-BB, M$\mathrm{BB}$, and $\mathrm{H}-\mathrm{BB})$ remarkably restored the locomotion time (23.2\%, $45.8 \%$, and $58.0 \%$ ) as compared to the PD model group (M) respectively $(P<0.05$; Table 1$)$. However, no significant effects on motor activity were observed in the sham-operated group treated with $20 \mathrm{mg} / \mathrm{kg}$ bilobalide as compared to the sham-operated group $(\mathrm{S})$.

Table 1. Effects of bilobalide pretreatment on motor activity in 6 OHDA-lesioned rats. No significant changes on motor activity were observed in sham-operated animals treated with $20 \mathrm{mg} / \mathrm{kg}$ bilobalide $(\mathrm{S}+\mathrm{H}-\mathrm{BB})$ as compared to the sham-operated group $(\mathrm{S})$. Locomotion time was significantly decreased in the PD model group (M). Different doses of bilobalide remarkably restored the locomotion time as compared to the $\mathrm{M}$ group, respectively. $n=6 .{ }^{\mathrm{b}} P<0.05$ vs $\mathrm{M}$

\begin{tabular}{lcc}
\hline & Bilobalide dose $\left(\mathrm{mg} \cdot \mathrm{kg}^{-1} \cdot \mathrm{d}^{-1}\right)$ & Locomotion time $(\mathrm{s})$ \\
\hline $\mathrm{S}$ & 0 & $240.50 \pm 9.75$ \\
$\mathrm{~S}+\mathrm{H}-\mathrm{BB}$ & 0 & $241.17 \pm 9.37$ \\
$\mathrm{M}$ & 0 & $55.50 \pm 6.15$ \\
$\mathrm{M}+\mathrm{L}-\mathrm{BB}$ & 5 & $111.33 \pm 8.27^{\mathrm{b}}$ \\
$\mathrm{M}+\mathrm{M}-\mathrm{BB}$ & 10 & $165.67 \pm 7.63^{\mathrm{b}}$ \\
$\mathrm{M}+\mathrm{H}-\mathrm{BB}$ & 20 & $196.67 \pm 6.94^{\mathrm{b}}$ \\
\hline
\end{tabular}

Bilobalide inhibited apomorphine-induced circling behavior The sham-operated animals failed to exhibit rotational behavior upon apomorphine challenge. The PD model rats exhibited contralateral rotations after the administration of apomorphine (430 \pm 40 turns/30 $\mathrm{min}$ ). Pretreatment with different doses of bilobalide significantly reduced contralateral rotations by a dose-dependent manner in the $\mathrm{M}+\mathrm{L}-\mathrm{BB}$, $\mathrm{M}+\mathrm{M}-\mathrm{BB}$, and $\mathrm{M}+\mathrm{H}-\mathrm{BB}$ groups, as compared to the $\mathrm{PD}$ model rats $(P<0.05$; Table 2$)$. Similar inhibitory effects of bilobalide on rotational behavior were observed at 2 and 3 weeks after 6-OHDA damage.

Table 2. Effects of bilobalide pretreatment on apomorphine-induced rotational behavior in 6-OHDA-lesioned rats. Rotational behavior was induced by a subcutaneous injection of apomorphine. No behavior change in the $\mathrm{S}$ and $\mathrm{S}+\mathrm{H}-\mathrm{BB}$ groups were observed. PD model group (M) showed significant contralateral rotations, while bilobalide reduced rotations in a dose-dependent manner. $n=6 .{ }^{\mathrm{b}} P<0.05$ vs $\mathrm{M}$.

\begin{tabular}{lccc}
\hline & $\begin{array}{c}\text { Bilobalide dose } \\
\left(\mathrm{mg} \cdot \mathrm{kg}^{-1} \cdot \mathrm{d}^{-1}\right)\end{array}$ & \multicolumn{2}{c}{ Rotation (circles/min) } \\
& & 2 weeks & 3 weeks \\
\hline $\mathrm{S}$ & 0 & $0.00 \pm 0.00$ & $0.00 \pm 0.00$ \\
$\mathrm{~S}+\mathrm{H}-\mathrm{BB}$ & 0 & $0.00 \pm 0.00$ & $0.00 \pm 0.00$ \\
$\mathrm{M}$ & 0 & $14.30 \pm 1.04$ & $14.56 \pm 1.02$ \\
$\mathrm{M}+\mathrm{L}-\mathrm{BB}$ & 5 & $5.86 \pm 0.98^{\mathrm{b}}$ & $6.13 \pm 1.28^{\mathrm{b}}$ \\
$\mathrm{M}+\mathrm{M}-\mathrm{BB}$ & 10 & $3.81 \pm 0.41^{\mathrm{b}}$ & $3.91 \pm 0.35^{\mathrm{b}}$ \\
$\mathrm{M}+\mathrm{H}-\mathrm{BB}$ & 20 & $2.96 \pm 0.41^{\mathrm{b}}$ & $3.13 \pm 0.62^{\mathrm{b}}$
\end{tabular}

Bilobalide reduced 6-OHDA-induced loss of dopaminergic neurons The loss of dopaminergic neurons in the SNpc was examined with TH immunofluorescence and Nissl's staining after 6-OHDA treatment. The infusion of 6-OHDA caused a rapid and consistent loss of $\mathrm{TH}$ immunoreactivity in the SNpc. By $24 \mathrm{~d}$ after 6-OHDA administration, a significant loss of TH immunoreactivity was observed. Pretreatment with bilobalide significantly reduced the 6-OHDA-induced loss of TH-positive neurons in the SNpc (Figure 1).

We extended our observations with Nissl's staining $24 \mathrm{~d}$ after 6-OHDA treatment. The results showed a remarkable loss of Nissl's body in the 6-OHDA-lesioned SNpc, and bilobalide substantially recovered the loss of nigral neurons (Figure 2).

Bilobalide blocked 6-OHDA-induced activation of NFкB NF- $\mathrm{BB}$ is known as an important transcriptional factor, playing a central role in the regulation of many immune and inflammatory responses, as well as the control of cell apoptosis. Recent evidence demonstrated the activation of $\mathrm{NF}-\kappa \mathrm{B}$ in neuronal cells during neurodegenerative processes. 
A

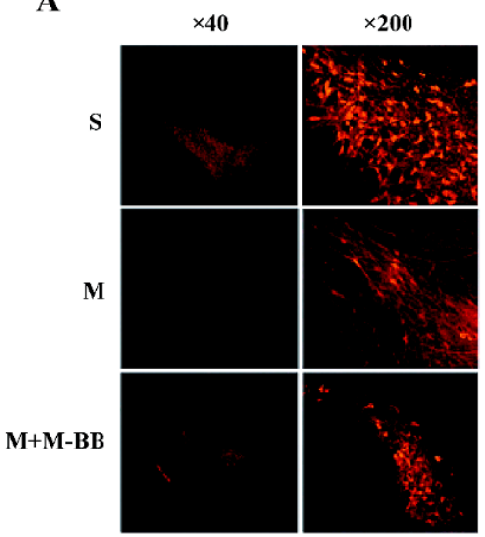

B

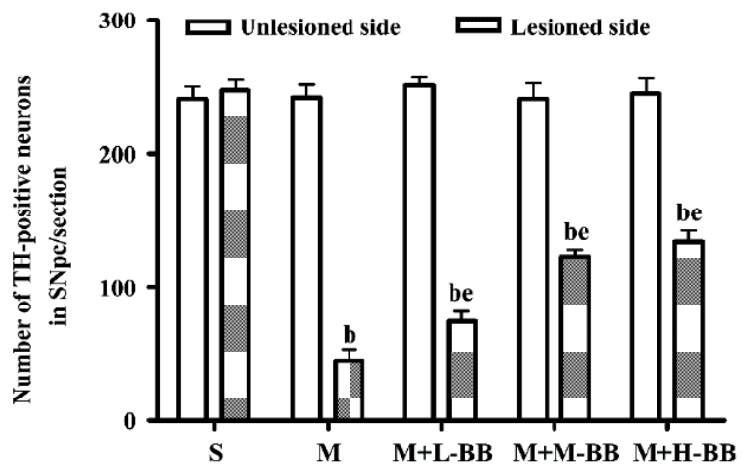

Figure 1. Bilobalide pretreatment reduced the loss of TH-positive neurons. Rats were unilaterally infused with 6-OHDA into the left SNpc and killed 24 d later. Brain sections were processed for TH immunofluorescence. TH-positive cells were counted with a fluorescence microscope. Number of TH-positive cells on the unlesioned and lesioned sides of the SN was obtained by averaging TH-positive cells in 6 brain sections from each animal. Results showed that pretreatment with different doses of bilobalide (5, 10, and $20 \mathrm{mg} / \mathrm{kg}$ ) for $7 \mathrm{~d}$ significantly reduced the loss of dopaminergic neurons induced by 6-OHDA. Red fluorescence shows TH-positive neurons in the SNpc (A). Each bar (B) represents the mean \pm SEM. $n=6$. ${ }^{\mathrm{b}} P<0.05$ vs unlesioned side; ${ }^{\mathrm{e}} P<0.05$ vs $\mathrm{M}$.

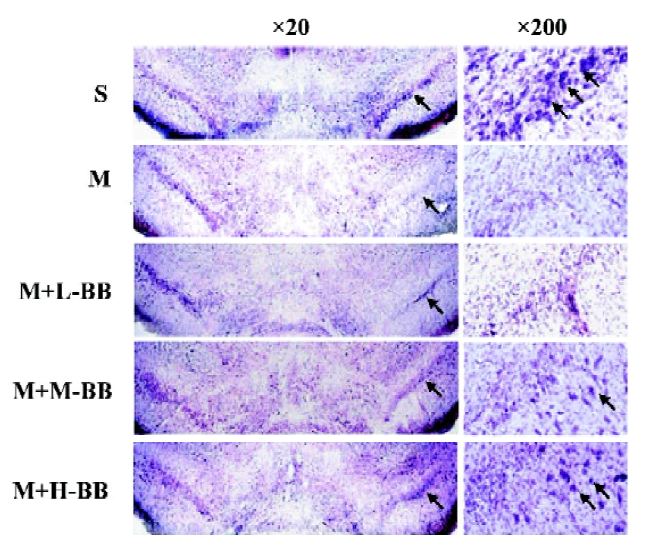

Figure 2. Bilobalide pretreatment reduced the loss of nigral cells. On d 24 of 6-OHDA injection, the rats were killed, and then the brain sections were processed for Nissl's staining to detect the neurons in the SNpc. Most neuronal somas on the left SNpc disappeared. Pretreatment with 10 or $20 \mathrm{mg} / \mathrm{kg}$ bilobalide increased the number of neurons in the lesioned SN. In the left graph $(\times 20)$, the violet lines indicated by black arrows show the SNpc. Right graph $(\times 200)$ shows the Nissl's body (black arrows).

The activation of NF-kB was examined in situ using immunofluorescence. The nuclear translocation of p65, a family member of NF- $\kappa \mathrm{B}$, was detected with immunofluorescence in dopaminergic neurons $24 \mathrm{~h}$ after 6-OHDA injection. The results showed that 6-OHDA induced a higher expression of NF- $\mathrm{KB}$ p65 in the nuclei of TH-positive neurons. Pretreatment with bilobalide at a dose of $10 \mathrm{mg}$ or higher effectively blocked 6-OHDA-induced NF- $\mathrm{BB}$ p65 nuclear translocation as revealed by immunofluorescence (Figure $3 \mathrm{~A})$. A quantitative analysis showed that the percentages of NF- $\kappa \mathrm{B}$ p65-positive dopaminergic neurons significantly decreased from $37.5 \% \pm 2.6 \%$ to $18.6 \% \pm 1.7 \%$ by pretreatment with bilobalide $(P<0.05$; Figure $3 \mathrm{~B})$.

Bilobalidereduced the numbersof terminaldeoxynucleotidyl transferase-mediated dUTP nick-end labeling-positive nuclei in the SNpc There were few terminal deoxynucleotidyl transferase-mediated dUTP nick-end labeling (TUNEL)-positive nuclei in the contralateral SN of PD model animals or in the ipsilateral SN of sham-operated animals. Increased numbers of TUNEL-positive nuclei were observed $24 \mathrm{~h}$ after 6OHDA infusion, which revealed apoptosis in the SNpc (Figure 4A). A quantitative analysis showed that the percentages of apoptosis in TH-positive neurons increased from $2.8 \% \pm 0.4 \%$ to $55.7 \% \pm 1.9 \%$ ( $P<0.05$; Figure $4 \mathrm{~B})$. We detected the co-expression of TUNEL and NF- $\kappa B$ p 65 nuclear translocation by double immunofluorescence. Elevated NF- $\mathrm{KB}$ p65 nuclear translocation and TUNEL-positive nuclei were observed $24 \mathrm{~h}$ after 6-OHDA damage (Figure 5). TUNEL and p65 staining were colocalized in the nuclei of the SNpc neurons. We found that pretreatment with bilobalide produced a significant decrease of NF- $\kappa$ B p 65 and TUNEL-positive nuclear staining.

\section{Discussion}

A number of reports indicate that $\mathrm{E} G b$ has wide pharmacological actions, such as anti-anemia ${ }^{[29]}$, anti-edema ${ }^{[30]}$, anti- 
$\mathbf{A}$

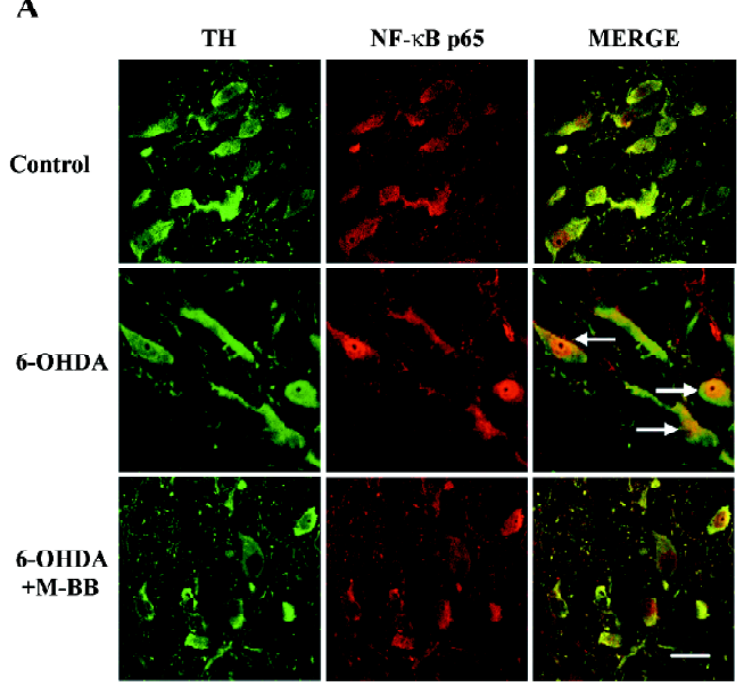

B

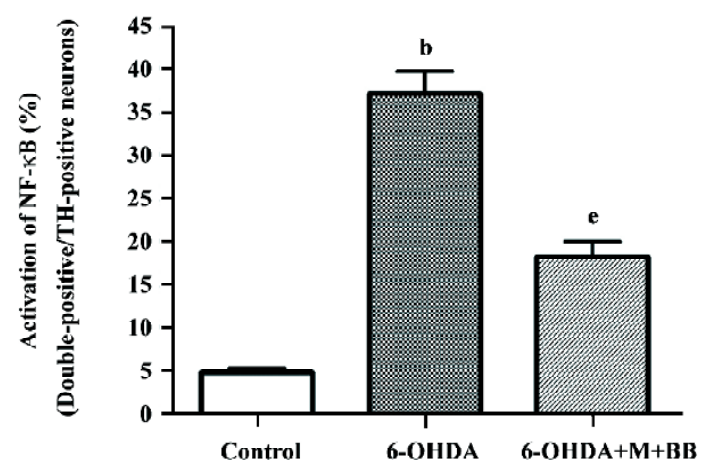

Figure 3. Pretreatment with bilobalide inhibited 6-OHDA-induced NF-KB p65 nuclear translocation in the SNpc. Rats were unilaterally infused with 6-OHDA into the left SNpc and killed $24 \mathrm{~h}$ later. Brain sections were processed with double immunofluorescence to test the nuclear translocation of NF-kB p65. (A) higher nuclear levels of NF$\kappa B$ p65 immunoreactivity (red) were seen in the TH-positive neurons (green). Pretreatment with bilobalide inhibited the nuclear translocation of NF-kB p65. (B) activation of NF- $\mathrm{kB}$ p65 in the dopaminergic neurons was evaluated by double-positive/TH-positive graphs. Data were expressed as mean \pm SEM. Experiments were repeated 3 times. ${ }^{\mathrm{b}} P<0.05$ vs control group. ${ }^{\mathrm{e}} P<0.05$ vs 6-OHDA group. Scale marker: $20 \mu \mathrm{m}$.

inflammation $^{[31]}$, anti-hypoxia ${ }^{[32]}$ and anti-oxidative stress ${ }^{[33]}$. Wettstein et $a l^{[34]}$ reported that EGb should be considered equally effective with second-generation cholinesterase inhibitors in the treatment of mild to moderate Alzheimer's dementia. Chandrasekaran et $a l^{[22]}$ reported that bilobalide exhibits protection against delayed ischemic neuronal death similar to that observed with the administration of ginkgo extracts. Consistent with this finding, Mdzinarishvili et al ${ }^{[35]}$ also demonstrated that bilobalide can prevent ischemia-in-

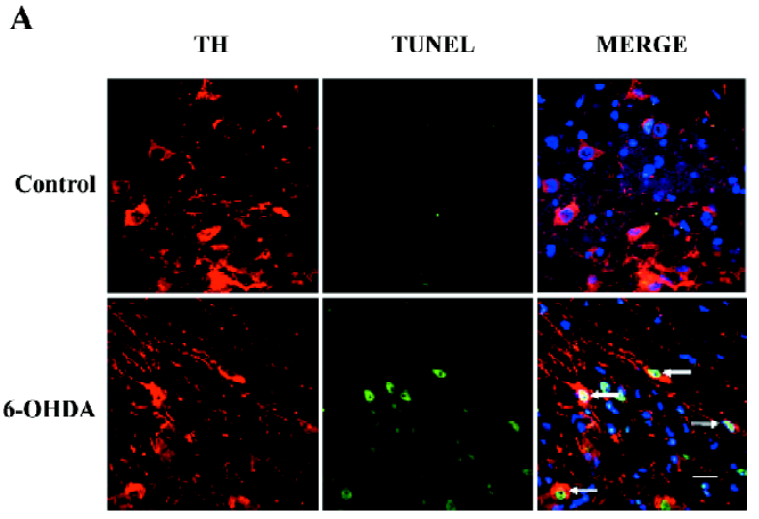

B

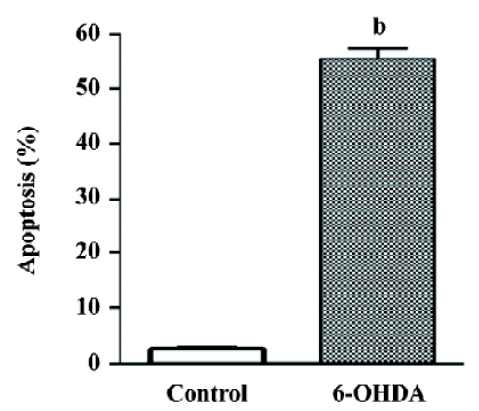

Figure 4. 6-OHDA-induced apoptosis of TH-positive neurons in the SNpc. Rats were unilaterally infused with 6-OHDA into the left SNpc and killed $24 \mathrm{~h}$ later. Brain sections were processed with TUNEL staining to detect the DNA damage of dopaminergic neurons, and the percentages of apoptosis were evaluated quantitatively. (A) TUNELpositive nuclei (green) were found in most dopaminergic neurons (TH-positive, red) in the 6-OHDA group (lower photo); nuclei were stained blue by DAPI. Several typical double-stained neurons are indicated by white arrows. (B) percentages of apoptotic neurons were accessed by double-positive/TH-positive graphs. Data were expressed as mean \pm SEM. Experiments were repeated 3 times. ${ }^{b} P<0.05$ vs control group. Scale marker: $20 \mu \mathrm{m}$.

duced edema formation in vitro and in vivo. Bilobalide could be an important active constituent of the extract. We attempted to find out whether bilobalide is able to inhibit neuronal necrosis and apoptosis induced by 6-OHDA in the $\mathrm{SNpc}$ of rats. Our study demonstrates that bilobalide can restore the locomotion, inhibit rotational behavior, and reduce loss of dopaminergic neurons, suggesting that bilobalide has neuroprotective effects and is able to improve the pathological symptoms in PD model rats.

At present, the mechanisms by which bilobalide protects neurons remain to be determined. In vitro and in vivo studies indicate that bilobalide has multiple actions that may be associated with neuroprotection, including preservation of mitochondrial ATP synthesis ${ }^{[36,37]}$, inhibition of apoptotic 


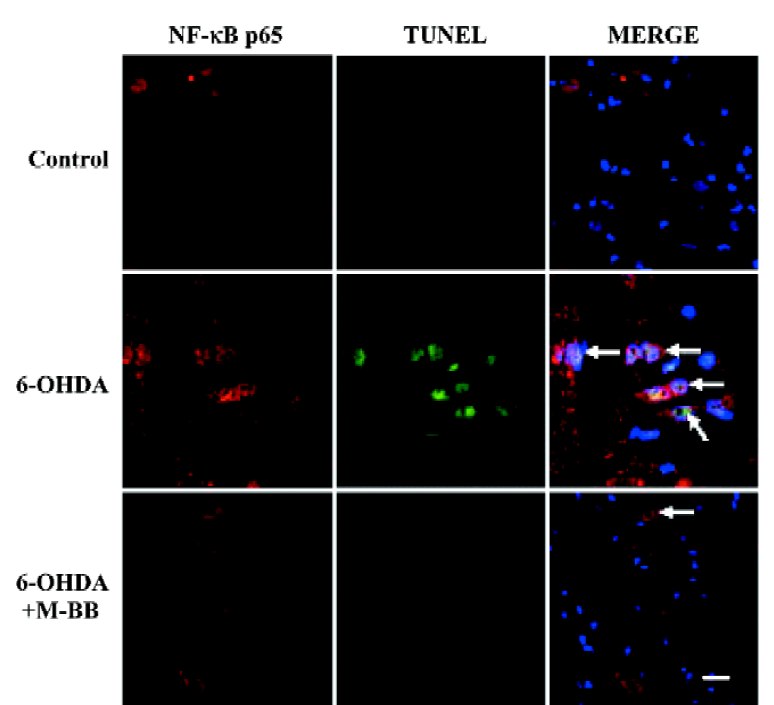

Figure 5. Bilobalide pretreatment decreased the colocalization of NF- $\mathrm{BB}$ p65 and TUNEL induced by 6-OHDA in the SNpc. Rats were unilaterally infused with 6-OHDA into the left SNpc and killed $24 \mathrm{~h}$ later. Increased number of TUNEL-positive nuclei (green), which co-expressed with NF- $\mathrm{BB}$ p65 (red), was seen in the SNpc after 6OHDA induced lesions. Nuclei were stained blue by DAPI. Bilobalide pretreatment significantly reduced the number of TUNEL-positive nuclei, as well as NF- $\mathrm{KB}$ p65 nuclear translocation. Scale marker: 20 $\mu \mathrm{m}$.

damage induced by staurosporine or by serum-free media ${ }^{[38]}$, suppression of hypoxia-induced membrane deterioration in the brain ${ }^{[39]}$, and regulation of mitochondrial gene expression $^{[23]}$. Zhou and $\mathrm{Zhu}^{[25]}$ demonstrated that bilobalide could attenuate ROS-induced apoptosis in PC12 cell lines, suggesting that bilobalide might be acting as a free-radical scavenger.

$\mathrm{NF}-\kappa \mathrm{B}$ appeared to be elevated in the $\mathrm{SN}$ of the postmortem brains of PD cases ${ }^{[40,41]}$. The relationship of NF- $\kappa B$ activation to the disease process, however, is unclear. Several in vitro studies have reported the activation of $\mathrm{NF}-\kappa \mathrm{B}$ in response to 6-OHDA treatment ${ }^{[42]}$. The intrastriatal administration of dopamine also produced oxidative damage to striatal neurons and a robust activation of $\mathrm{NF}-\kappa \mathrm{B}^{[43]}$, but evaluations of the role of $\mathrm{NF}-\kappa \mathrm{B}$ in in vitro $\mathrm{PD}$ models have not yielded consistent results ${ }^{[44,45]}$. Some studies reported that the neuroprotective effect offered by some pharmacological agents is associated with the blockade of NF- $\kappa \mathrm{B}$ activation, suggesting that NF- $\kappa \mathrm{B}$ plays a pro-apoptotic role in $\mathrm{PD}^{[46-48]}$. In our previous study, 6-OHDA induced an increase in the binding activity of NF- $\kappa \mathrm{B}$, which provided the first biochemical evidence of the activation of NF- $\mathrm{KB}$ in animal models of PD.
In this study, 6-OHDA-induced apoptosis of dopaminergic neurons was accompanied by NF- $\kappa \mathrm{B}$ activation. It was implied that the activation of NF- $\kappa \mathrm{B}$ p 65 contributed to the apoptosis of nigral neurons. A decrease in total cell numbers and increase in apoptotic cells induced by 6-OHDA were significantly attenuated by bilobalide pretreatment. NF$\kappa \mathrm{B}$ activation and an increase in DNA fragmentation induced by 6-OHDA were also significantly inhibited. Bilobalide may block apoptosis of dopaminergic neurons through the suppression of the expression of the NF- $\mathrm{KB}$ p65 protein and decrease its nuclear translocation in the SNpc of rats.

In summary, the present study shows that apoptosis is involved in 6-OHDA-induced dopaminergic neuronal death, and that the activation of NF- $\kappa$ B plays an important role in apoptosis. Pretreatment with bilobalide effectively prevents the activation of NF- $\mathrm{KB}$ and produces a marked protective effect on dopaminergic neurons against the toxicity induced by $6-\mathrm{OHDA}$ in the SNpc. Bilobalide may thereby provide a therapeutic approach to rescue the dopaminergic neurons in the process of PD.

\section{Acknowledgment}

Bilobalide was provided by Prof Zhong-liang CHEN from the Department of Phytochemistry, Shanghai Institute of Materia Medica, Chinese Academy of Sciences (Shanghai, China).

\section{References}

1 Chinta SJ, Andersen JK. Dopaminergic neurons. Int J Biochem Cell Biol 2005; 37: 942-6.

2 Ziv I, Barzilai A, Offen D, Nardi N, Melamed E. Nigrostriatal neuronal death in Parkinson's disease--a passive or an active genetically-controlled process? J Neural Transm Suppl 1997; 49: 69-76.

3 Barzilai A, Melamed E, Shirvan A. Is there a rationale for neuroprotection against dopamine toxicity in Parkinson's disease? Cell Mol Neurobiol 2001; 21: 215-35.

4 Qin ZH, Wang Y, Nakai M, Chase TN. Nuclear factor- $\kappa B$ contributes to excitotoxin-induced apoptosis in rat striatum. Mol Pharmacol 1998; 53: 33-42.

5 Qin ZH, Chen RW, Wang Y, Nakai M, Chuang DM, Chase TN. Nuclear factor $\kappa B$ nuclear translocation upregulates c-Myc and p53 expression during NMDA receptor-mediated apoptosis in rat striatum. J Neurosci 1999; 19: 4023-33.

6 Liang ZQ, Li YL, Zhao XL, Han R, Wang XX, Wang Y, et al. NF- $\kappa \mathrm{B}$ contributes to 6-hydroxydopamine-induced apoptosis of nigral dopaminergic neurons through p53. Brain Res 2007; 1145 : 190-203.

7 Panet H, Barzilai A, Daily D, Melamed E, Offen D. Activation of nuclear transcription factor $\kappa \mathrm{B}(\mathrm{NF}-\kappa \mathrm{B})$ is essential for dopamine-induced apoptosis in PC12 cells. J Neurochem 2001; 77: 391-8. 
8 DeFeudis FV, Drieu K. Ginkgo biloba extract (EGb 761) and CNS functions: basic studies and clinical applications. Current Drug Targets 2000; 1: 25-58.

9 Ahlemeyer B, Krieglstein J. Neuroprotective effects of Ginkgo biloba extract. Cell Mol Life Sci 2003; 60: 1779-92.

10 Ahlemeyer B, Krieglstein J. Pharmacological studies supporting the therapeutic use of Ginkgo biloba extract for Alzheimer's disease. Pharmacopsychiatry 2003; 36 (Suppl 1): S8-14.

11 Grundman M, Grundman M, Delaney P. Antioxidant strategies for Alzheimer's disease. Proc Nutr Soc 2002; 61: 191-202.

12 DeFeudis FV. Ginkgo biloba extract (EGb761): Pharmacological activities and clinical applications. Paris: Elsevier; 1991.

13 Zhang J, Fu S, Liu S, Mao T, Xiu R. The therapeutic effect of Ginkgo biloba extract in SHR rats and its possible mechanisms based on cerebral microvascular flow and vasomotion. Clin Hemorheol Microcirc 2000; 23: 133-8.

14 Tchantchou F, Xu Y, Wu Y, Christen Y, Luo Y. EGb 761 enhances adult hippocampal neurogenesis and phosphorylation of CREB in transgenic mouse model of Alzheimer's disease. FASEB J 2007; 21: 2400-8.

15 Brunetti L, Orlando G, Menghini L, Ferrante C, Chiavaroli A, Ginkgo biloba leaf extract reverses amyloid beta-peptide-induced isoprostane production in rat brain in vitro. Planta Med 2006; 72: 1296-9.

16 Pardon MC, Joubert C, Perez-Diaz F, Christen Y, Launay JM, Cohen-Salmon C. In vivo regulation of cerebral monoamine oxidase activity in senescent controls and chronically stressed mice by long-term treatment with Ginkgo biloba extract (EGb 761). Mech Ageing Dev 2000; 113: 157-68.

$17 \mathrm{Wu}$ WR, Zhu XZ. Involvement of monoamine oxidase inhibition in neuroprotective and neurorestorative effects of Ginkgo biloba extract against MPTP-induced nigrostriatal dopaminergic toxicity in C57 mice. Life Sci 1999; 65: 157-64.

18 Ahmad M, Saleem S, Ahmad AS, Yousuf S, Ansari MA, Khan MB, et al. Ginkgo biloba affords dose-dependent protection against 6hydroxydopamine-induced parkinsonism in rats: neurobehavioural, neurochemical and immunohistochemical evidences. J Neurochem 2005; 93: 94-104.

19 Cao F, Sun S, Tong ET. Experimental study on inhibition of neuronal toxical effect of levodopa by Ginkgo biloba extract on Parkinson's disease in rats. J Huazhong Univ Sci Tech Med Sci 2003; 23: 151-3.

20 Otani M, Chatterjee SS, Gabard B, Kreutzberg GW. Effect of an extract of Ginkgo biloba on triethyltin-induced cerebral edema. Acta Neuropathol 1986; 69: 54-65.

21 Chandrasekaran K, Mehrabian Z, Spinnewyn B, Drieu K, Fiskum G. Neuroprotective effects of bilobalide, a component of the Ginkgo biloba extract (EGb 761), in gerbil global brain ischemia. Brain Res 2001; 922: 282-92.

22 Chandrasekaran K, Mehrabian Z, Spinnewyn B, Chinopoulos C, Drieu K, Fiskum G. Bilobalide, a component of the Ginkgo biloba extract (EGb 761), protects against neuronal death in global brain ischemia and in glutamate-induced excitotoxicity. Cell Mol Biol 2002; 48: 663-9.

23 Chandrasekaran K, Mehrabian Z, Spinnewyn B, Chinopoulos C, Drieu K, Fiskum G. Neuroprotective effects of bilobalide, a component of Ginkgo biloba extract (EGb 761) in global brain ischemia and in excitotoxicity-induced neuronal death. Pharmacopsychiatry
2003; 36 (Suppl 1): S89-94.

24 Ahlemeyer B, Krieglstein J. Neuroprotective effects of Ginkgo biloba extract. Cell Mol Life Sci 2003; 60: 1779-92.

25 Zhou LJ, Zhu XZ. Reactive oxygen species-induced apoptosis in PC12 cells and protective effect of bilobalide. J Pharmacol Exp Ther 2000; 293: 982-8.

26 Weichel O, Hilgert M, Chatterjee SS, Lehr M, Klein J. Bilobalide, a constituent of Ginkgo biloba, inhibits NMDA-induced phospholipase $\mathrm{A}_{2}$ activation and phospholipid breakdown in rat hippocampus. Naunyn Schmiedebergs Arch Pharmacol 1999; 360: 609-15.

27 Carman LS, Gage FH, Shults CW. Partial lesion of the substantia nigra: relation between extent of lesion and rotational behavior. Brain Res 1991; 553: 275-83.

28 West MJ, Slomianka L, Gundersen HJ. Unbiased stereological estimation of the total number of neurons in the subdivisions of the rat hippocampus using the optical fractionator. Anat Rec 1991; 231: 482-97.

29 Sarikcioglu SB, Oner G, Tercan E. Antioxidant effect of EGb 761 on hydrogen peroxide-induced lipoperoxidation of G-6-PD deficient erythrocytes. Phytother Res 2004; 8: 837-40.

30 Biddlestone L, Corbett AD, Dolan S. Oral administration of Ginkgo biloba extract, EGb-761 inhibits thermal hyperalgesia in rodent models of inflammatory and post-surgical pain. $\mathrm{Br} \mathrm{J}$ Pharmacol 2007; 151: 285-91.

31 Han Y. Ginkgo terpene component has an anti-inflammatory effect on Candida albicans-caused arthritic inflammation. Int Immunopharmacol 2005; 5: 1049-56.

32 Sharma HS, Drieu K, Westman J. Antioxidant compounds EGb761 and BN-52021 attenuate brain edema formation and hemeoxygenase expression following hyperthermic brain injury in the rat. Acta Neurochir Suppl 2003; 86: 313-9.

33 Smith JV, Luo Y. Elevation of oxidative free radicals in Alzheimer's disease models can be attenuated by Ginkgo biloba extract EGb 761. J Alzheimers Dis 2003; 5: 287-300.

34 Wettstein A. Cholinesterase inhibitors and Gingko extracts-are they comparable in the treatment of dementia? Comparison of published placebo-controlled efficacy studies of at least six months' duration. Phytomedicine 2000; 6: 393-401.

35 Mdzinarishvili A, Kiewert C, Kumar V, Hillert M, Klein J. Bilobalide prevents ischemia-induced edema formation in vitro and in vivo. Neuroscience 2007; 144: 217-22.

36 Janssens D, Delaive E, Remacle J, Michiels C. Protection by bilobalide of the ischaemia-induced alterations of the mitochondrial respiratory activity. Fundam Clin Pharmacol 2000; 14: 193-201.

37 Bouaziz N, Redon M, Quere L, Remacle J, Michiels C. Mitochondrial respiratory chain as a new target for anti-ischemic molecules. Eur J Pharmacol 2002; 441: 35-45.

38 Ahlemeyer B, Möwes A, Krieglstein J. Inhibition of serum deprivation- and staurosporine-induced neuronal apoptosis by Ginkgo biloba extract and some of its constituents. Eur J Pharmacol 1999; 367: 423-30.

39 Klein J, Chatterjee SS, Loffelholz K. Phospholipid breakdown and choline release under hypoxic conditions: inhibition by bilobalide, a constituent of Ginkgo biloba. Brain Res 1997; 755: $347-50$

40 Hunot S, Brugg B, Ricard D, Michel PP, Muriel MP, Ruberg M, et 
al. Nuclear translocation of NF- $\mathrm{KB}$ is increased in dopaminergic neurons of patients with Parkinson disease. Proc Natl Acad Sci USA 1997; 94: 7531-6.

41 Soos J, Engelhardt JI, Siklos L, Havas L, Majtenyi K. The expression of PARP, NF- $\kappa \mathrm{B}$ and parvalbumin is increased in Parkinson's disease. Neuroreport 2004; 15: 1715-8.

42 Tarabin V, Schwaninger $M$. The role of $\mathrm{NF}-\kappa \mathrm{B}$ in 6 hydroxydopamine- and TNF $\alpha$-induced apoptosis of PC12 cells. Naunyn Schmiedebergs Arch Pharmacol 2004; 369: 563-9.

43 Lou Y, Hattori A, Munoz J, Qin ZH, Roth GS. Intrastriatal dopamine injection induces apoptosis through oxidation-involved activation of transcription factors AP-1 and NF- $\mathrm{KB}$ in rats. Mol Pharmacol 1999; 56: 254-64.

44 Panet H, Barzilai A, Daily D, Melamed E, Offen D. Activation of nuclear transcription factor $\kappa \mathrm{B}(\mathrm{NF}-\kappa \mathrm{B})$ is essential for dopamine-induced apoptosis in PC12 cells. J Neurochem 2001; 77: 391-8.
45 Park SH, Choi WS, Yoon SY, Ahn YS, Oh YJ. Activation of NF$\kappa \mathrm{B}$ is involved in 6-hydroxydopamine- but not MPP+-induced dopaminergic neuronal cell death: its potential role as a survival determinant. Biochem Biophys Res Commun 2004; 322: 72733.

46 Dehmer T, Heneka MT, Sastre M, Dichgans J. Protection by pioglitazone in the model of Parkinson's disease correlates with $\mathrm{I} \kappa \mathrm{B} \alpha$ induction and block of NF- $\mathrm{KB}$ and iNOS activation. J Neurochem 2004; 88: 494-501.

47 Levites Y, Youdim BH, Maor G, Mandel S. Attenuation of 6hydroxydopamine (6-OHDA)-induced nuclear factor-kappaB $(\mathrm{NF}-\kappa \mathrm{B})$ activation and cell death by tea extracts in neuronal cultures. Biochem Pharmacol 2002; 63: 21-9.

48 Youdim MB, Grunblatt E, Mandel S. The pivotal role of iron in $\mathrm{NF}-\kappa \mathrm{B}$ activation and nigrostriatal dopaminergic neurodegeneration. Prospects for neuroprotection in Parkinson's disease with iron chelators. Ann N Y Acad Sci 1999; 890: 7-25.

\title{
the $4^{\text {th }}$ Hong Kong-Shanghai International Liver Congress (ILC) 2008
}

\author{
Hong Kong, China \\ June $12-15,2008$
}

for more information, please contact

Congress Secretariat:

Gary Ng / Lokman Wong

9/F, New Hennessy Tower

263 Hennessy Road, Wan Chai,

$$
\text { Hong Kong }
$$

Phn 852-2827-2090

Fax $\quad 852-2827-2220$

Email:info@cosoman.com

http://www.livercongress.org 